\title{
Isolated ano-perianal tuberculosis: challenging diagnosis
}

\begin{abstract}
The tuberculosis is a public health issue. The ano -perianal localization is very rare, representing less than $0.7 \%$ of the digestive localizations. Basing on personal observation and literature review, we cite the diagnostic difficulties and the epidemic clinical, therapeutic and evolutive particularities of this rare entity. A 34year-old-women was admitted for the exploration of chronic suppuration of the perianal and gluteal region which has been in progress since 10years. Many investigations were made and we retained the diagnosis of ano-perianal tuberculosis localization after detecting the presence of Koch bacillus on Lowenstein Jensen culture of biopsies and stools. The patient was treated with Quadratherapy. Our observation illustrates the difficulties in diagnosing ano-perineal tuberculosis with chronic anal suppuration causing a delayed diagnosis and therapeutic management.
\end{abstract}

Keywords: ano-perineal tuberculosis, chronic suppuration, external orifices of the fistulas, lowenstein jensen culture, koch bacillus
Volume 10 Issue 5 - 2019

\begin{abstract}
Boudabous M, Gdoura H, Chtourou L, Amouri A, Mnif L, Tahri N

Department of Hepato-gastroenterology service, University of sfax, Tunisia
\end{abstract}

Correspondence: Boudabous Mona, Department of Hepatogastroenterology service, University of sfax, Tunisia, Email boudabousmona@yahoo.fr

Received: September 12, 2019 | Published: October I, 2019

\section{Introduction}

The tuberculosis is a public health issue and a granulomatous disease caused by Mycobacterium tuberculosis. The extra pulmonary dissemination represents less than $15 \%$ of the total cases of tuberculosis. ${ }^{1,2}$ while the intestinal dissemination represents only less than $1 \%{ }^{3,4}$ The ano-perianal localization is very rare, representing less than $0.7 \%$ of the digestive localizations..$^{3-6}$ and can take different forms having the ability to mime other pathologies. It can be associated with an abdominal tuberculosis either through the extension from an adjacent organ or the lymphatic canal. Basing on personal observation and literature review, we cite the diagnostic difficulties and the epidemic clinical, therapeutic and evolutive particularities of this rare entity.

\section{Observation}

A patient, aged of 43 years old, without any particular previous pathologies, was admitted for the exploration of chronic suppuration of the perianal and gluteal region which has been in progress since 10years without any troubles of transit or signs of tuberculosis impregnations. The patient was operated, one year ago, for anal abscess. But she had noted a quick recidivism of the suppuration and a multiplication of the external orifices of the fistulas. The examination of the anal margin had revealed 4 productive orifices surrounded by a sclerotic and carded skin. The hemoglobin level was $9 \mathrm{~g}$ per $\mathrm{dl}$ (microcytic hypochromic anemia) and there was an inflammatory syndrome. The serology HIV was negative. Rectoscopy revealed two internal orifices of the fistula. The ileocoloscopy was normal. The thoraco-abdominal scanner was normal. The pelvic MRI revealed extra-sphincterial multiple anal fistulas (Figure 1). Tuberculin intradermal reaction was positive at $15 \mathrm{~mm}$. The anatomopathological examination of the skin biopsies of the gluteal region had lead to a granulomatous dermatitis of the anal region without caseous necrosis. The direct bacteriological examination as well as the BK PCR of these biopsies was negative. One month after these samples were taken, Lowenstein Jensen culture of these biopsies and stools showed the presence of BK, which is sensitive to the usual anti-tuberculosis drugs. The patient was treated with Quadratherapy (rifampycin-isoniazid-ethambutol-pyrazinamide) for 2 months followed by 4 months of dual therapy (Rifampycin isoniazid). The outcome was favorable with drying of the fistulas and disappearance of the inflammatory syndrome as early as 30day of treatment (Figure 2) without recurrence after discontinuation of the treatment with a current follow-up of 4,5years.

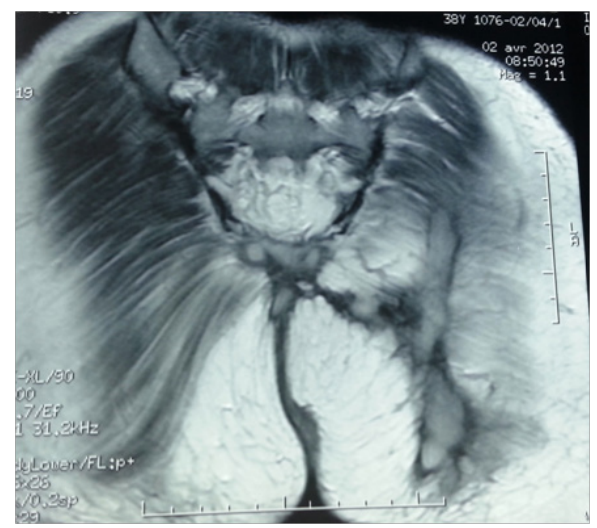

Figure I Pelvic MRI revealed extra-sphincterial anal fistula.

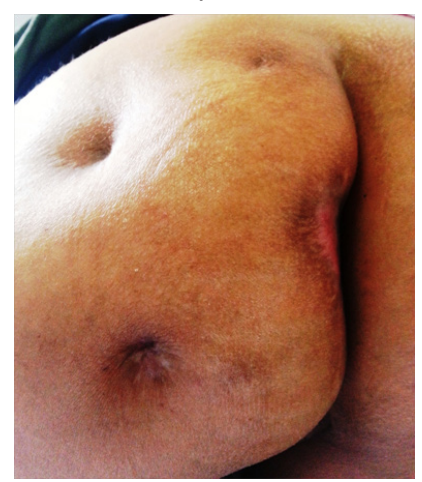

Figure 2 Drying of the fistulas after 30 days of treatment. 


\section{Discussion}

Our observation illustrates the difficulties in diagnosing anoperineal tuberculosis with chronic anal suppuration causing a delayed diagnosis and therapeutic management. Actually, it was only after 10years of evolution and the failure of a surgical cure that the diagnosis of tuberculosis was suspected in our patient. Three factors cause these difficulties:

a. the scarcity of isolated anoperineal tuberculosis,

b. the low sensitivity of diagnostic tests destined to confirm anoperineal tuberculosis,

c. and the presence of multiple other diseases that can cause chronic anal suppuration and pose problems of differential diagnosis with anoperineal tuberculosis.

The frequency of isolated ano-perineal tuberculosis is very rare, representing less than $0.7 \%$ of digestive tracts, which constitute only $1 \%$ of extra-pulmonary tuberculosis. ${ }^{3,4}$ Ano-perineal tuberculosis is more common in men than in women (sex ratio 4/1). It is diagnosed generally in the fourth decade as the case of our patient. The contamination of the ano-perineal region can be done via the digestive tract (direct ingestion of pulmonary secretions infected with the Koch bacillus or contaminated food), through the blood circulation with a pulmonary starting point, trough the lymphatic canals from the lymph nodes Infected or by contiguity from the adjacent affected organs. ${ }^{5,7,8}$

Despite the variability of diagnostic tests, the diagnosis of tuberculosis remains a challenge for the clinician. Tuberculin intradermal reaction is not very specific. ${ }^{9}$ It is generally positive in $70 \%$ of cases but may be negative in cases of immunosuppression, malnutrition or in the case of associated anergizing disease such as sarcoidosis. ${ }^{10,11}$ Tests for the production of Interferon $\gamma$ by Tlymphocytes sensitized to Mycobacterium hominis, in particular Quantiféron ${ }^{\circledR}$ and Elispot-TB ${ }^{\circledR}$, are incompletely sensitive and very specific to the presence of Mycobacterium hominis living in the organism, ${ }^{13}$ but do not allow the distinction between latent and evolutionary tuberculosis. When the result of the test is said to be indeterminate, it means that the T lymphocytes of the patient are unable (in number or function) to produce interferon in the presence of control mitogens. In this case, the test does not contribute to the diagnosis of tuberculosis either way. ${ }^{12}$ Koch bacillus's research in sputum, chest X-ray and thoracic CT scan are routinely performed in search of a possible pulmonary outbreak, but pulmonary involvement is found in less than $50 \%$ of patients with perineal tuberculosis. ${ }^{13}$ The formal diagnosis is based on histological or bacteriological arguments. The typical histological lesion is the association of an epithelioid and giganto-cellular granuloma with caseous necrosis. ${ }^{13}$ The identification of the Koch bacillus is carried out either by direct examination after Ziehl-Neelsen staining. ${ }^{13}$ or after culture which constitutes a reference method with a sensitivity of 60 to $90 \%$ and a specificity of $100 \%$ allowing the diagnosis and conducting an antibiogram. It can be done in solid media called « Lowenstein Jensen' media" that require 3 to 6 weeks incubation period or in liquid medium having as advantage a quick result in 2 to 8 days but at the price of a higher risk of contamination. ${ }^{13,16,17}$ This technique allowed us to make the diagnosis in our patient. The diagnosis can also be carried out by molecular biology which allows the identification of mycobacteria of the tuberculosis complex via the determination of the biochemical or cultural characteristics. ${ }^{11}$ In particular, the gene amplification technique (PCR), which detects and amplifies a specific nucleic sequence of the $\mathrm{M}$. tuberculosis complex has a high specificity but does not allow the distinction between dead BK and living BK, does not provide information on the degree of Contagion and does not recognize certain existing mutations. ${ }^{12}$ The GeneXpert MTB / RIF, automated real-time PCR, detects the presence of mycobacterium tuberculosis with a rapid result and a high specificity and allows the diagnosis of extra pulmonary tuberculosis especially through biopsies (sensitivity $77.3 \%$, specificity $98.2 \%$ ). ${ }^{12,14}$ Yet, our patient's test was negative.

The diagnosis of isolated perineal tuberculosis is difficult not only because of its rarity or the low sensitivity of the tests for diagnostic purposes, but also because of the presence of other differential diagnoses, which are sometimes difficult to eliminate. Crohn's disease, in particular, constitutes a major differential diagnosis, the distinction of which with perineal tuberculosis constitutes a real challenge for the clinician..$^{15}$ Indeed, these two pathologies have common points as well on the clinical, morphological and histological level. In $1 / 3$ to $1 / 2$ of cases, anoperineal lesions inaugurate Crohn's disease. ${ }^{15}$ In particular, ano-perineal involvement can be isolated during Crohn's disease and include giganto-cellular granulomas on the histological examination of biopsies but without caseous necrosis. For this reason, we could not initially rule out the diagnosis of Crohn's disease in our patient despite the presence of granulomatous dermatitis in the skin biopsies of the gluteal region. In these difficult situations, histological features of the granulomas can be helpful. Indeed, tuberculous granulomas are typically larger than those of MC, sit mainly under ulcerations and are readily confluent and absent in non-inflammatory mucosa. In addition, indirect arguments from the context could tip the balance towards the diagnosis of tuberculosis, such as the presence of endemic areas, familial or occupational contagion, Tubertest ${ }^{\circledR}$ (5units) positive (Induration $>4 \mathrm{~mm}$ ) without a history of BCG vaccine or very positive $(>9 \mathrm{~mm})$ at a distance (>10years) of a BCG vaccine (found in our patient) or a positive Quantiferon. ${ }^{16-18}$ Other diseases may also induce chronic anoperineal lesions, such as Verneuil's disease, which is a chronic inflammatory and suppurative disease developing on skin territories with apocrine sweat glands. During this disease, the anoperineal localization, representing $20 \%$ of the cases, is more frequent in the man than in the woman. It is characterized by the appearance of a nodule that evolves towards suppuration and the formation of abscesses that can break up, giving way to productive fistulous orifices. ${ }^{19,20}$ In addition to Verneuil's disease, some infections may lead to suppurative anoperineal lesions such as lymphogranuloma venereum or Nicolas Favre's disease, syphilis and amoebiasis. ${ }^{5}$ Venereal lymphogranuloma, due to Chlamydia Trachomatis, may be the cause of condylomata, ulcerations and oozing fistulous pathways. Its diagnosis is based on the search for Chlamydia Trachomatis DNA by PCR after swabbing. ${ }^{21}$ Syphilis from Treponema pallidum is manifested in its primary phase by lateralized ulcerations associated with inguinal unilateral lymphadenopathy, but some atypical forms may be the cause of chronic anal fissures, oozing raghades or multiple ulcerations. The diagnosis is carried out by demonstrating the agent responsible under a dark-field microscope after sampling at lesions or by TPHA-VDRL serology. ${ }^{21,22}$ Perianal amoebiasis, occurring in male homosexuals after oro-anal intercourse, is manifested by anal ulcerations of painful variable sizes with an indurated base, in which an amoebic rectitium facilitates diagnosis in the classical forms. ${ }^{23}$ Tumor proliferation also constitutes a differential diagnosis which must be eliminated in principle by a good histopathological analysis. Indeed, adenocarcinoma of the anal margin may occur on a chronic anorectal fistula. ${ }^{24}$ As for pulmonary localization, the treatment of anoperineal tuberculosis is based on anti-tuberculosis drugs for a period of six months. The evolution is generally favorable as it is the case for our patient. ${ }^{25,26}$ Surgery in the case of fistulae may be 
recommended in combination with medical treatment in order to get good drainage of infected lesions. A second surgical time is discussed after well-conducted anti-tuberculosis treatment, depending on the height and number of fistulous pathways. ${ }^{27}$ Surgery was not necessary for our patient.

\section{Conclusion}

To conclude, we report a rare case of isolated anoperineal tuberculosis revealed by chronic suppuration of the anoperineal region. It illustrates the difficulties of positive and differential diagnosis and specifies the principles of the management of this rare entity.

\section{Acknowledgments}

None.

\section{Conflict of interest}

Author declares there are no conflicts of interest.

\section{References}

1. Mylarappa P, Kudva A, Rao A. Perianal tuberculosis. Indian J Tuber. 1997;44(4):209-210.

2. Candela F, Serrano P, Arriero JM, et al. Perianal disease of tuberculous origin: report of a case and review of the literature. Dis Colon Rectum. 1999;42(1):110-112.

3. Bravo FG, Gotuzz E. Cutaneous tuberculosis. Clin Dermatol. 2007;25(2):173-180.

4. Yadegarynia D, Abbasi F, Keshtkar-Jahromi M, Gholamin S Gastrointestinal tuberculosis with cecum involvement in a 33-year-old woman. Iran J Med Sci. 2009;34(3):213-216.

5. Mosena G, Della Giustina A, Matos D’Almeida Santos JC, et al. Perianal tuberculosis in an immunocompetent patient. J Eur Acad Dermatol Venereol. 2018;32(6):e229-e230.

6. Tago S, Hirai Y, Ainoda Y, et al. Perianal tuberculosis: A case report and review of the literature. World J Clin Cases. 2015;3(9):848-852.

7. Ghosh SK, Bandyopadhyay D, Ghosh A, et al. Non-healing perianal ulcer: A rare presentation of cutaneous tuberculosis. Dermatol Online J. 2009;15(3):9.

8. Tai WC, Hu TH, Lee $\mathrm{CH}$, et al. Ano-perianal tuberculosis, 15years of clinical experiences in Southern Taiwan. Colorectal Dis. 2010;12(7):e114e120.

9. Shingadia D, Novelli V. The tuberculin skin test: a hundred, not out? Arch Dis Child. 2008;93(3):189-190.
10. Black GF, Dockrell HM, Crampin AC, et al. Patterns and implications of naturally acquired immune responses to environmental and tuberculous mycobacterial antigens in northern Malawi. J Infect Dis. 2001;184(3):322329 .

11. Garg SK, Tiwari RP, Tiwari D, et al. Diagnosis of tuberculosis: available technologies, limitations and possibilities. J Clin Lab Anal. 2003;17(5):155e63.

12. Piersmoni C. Current perspectives on drug susceptibilty testing of Mycobacterium tuberculosis complex: the automated non-radiometric sys-tems. J Clin Microbiol. 2006;44(1):20-28.

13. Sultan S, Azria F, Bauer P, et al. Anoperineal tuberculosis: diagnostic and management considerations in seven cases. Dis Colon Rectum. 2002;45(3):407-410.

14. Uribe-Bojanini E, et al. Patient with disseminated tuberculosis and rare perianal involvement. Actas Dermosifiliogr. 2016 ;107(10):874-876.

15. Boehme C. Rapid molecular detection of tuberculosis and rifampicin resistance. N Engl J Med. 2010;363:1005-1015.

16. Beaugerie L. Initial diagnosis of IBD: diagnostic criteria and diagnosis differential. MICU 201-2016.

17. Baumgart D, Sandborn W. Crohn's disease. Lancet. 2012;3880:15901605.

18. Loh KW, Bassily R, Torresi J. Crohn's disease or tuberculosis? J Travel Med. 2011;18:221-223.

19. Sénejoux A. Verneuil ano-perineal disease. POST'U. 2014;1-4.

20. Dhaou BB, BoussemaF, Aydi Z. Hidradenitis suppurativa (Verneuil's disease). Journal of the Saudi Society of Dermatology \& Dermatologic Surgery. 2013;17(1):1-5.

21. John A. Lymphogranuloma venereum (LGV). Medicine. 2014;42(7):399402.

22. Sukthankar A. Syphilis. Medicine. 2014;42(7):394-398.

23. Wright S. Amoebiasis and giardiasis. Medicine. 2005;33(8):47-50.

24. Gaertner WB, Hagerman GF, Finne CO, et al. Fistula-associated anal adenocarcinoma: good results with aggressive therapy. Dis Colon Rectum. 2008;51(7):1061-1067.

25. World Health Organization (WHO). Third Global report. Global project on antituberculosis drug resistance surveillance. Geneva: World Health Organization; 2004.

26. World Health Organization (WHO). Treatment of tuberculosis: guidelines for national programmes. 4th edn. Geneva: World Health Organization; 2003. 